\title{
How Stigma Gets Under the Skin: Internalized Oppression and Dual Minority Stress among Black Sexual Minorities
}

\author{
Alex A. Ajayi and Moin Syed \\ University of Minnesota
}

\begin{abstract}
The purpose of this chapter is to examine the links between oppressive social conditions and psychological well-being among Black sexual minorities. In particular, we examine the nature and process by which members of marginalized groups may come to internalize the negative messages about their nondominant social identity (i.e., internalized oppression). Given that Black sexual minorities are simultaneously subjugated to systems of oppression based on their race and their sexuality, they will experience what we have termed "dual minority stress". By examining the narratives of 15 Black sexual minorities, we provide empirical support for the dual minority stress model and shed light on the characteristics of internalized oppression and the social conditions by which oppression is internalized. Specifically, our findings point to three important manifestations of internalized oppression: psychological maladjustment, acting-out mechanisms, and identity disintegration. We also identify three mechanisms by which oppression becomes internalized: notions of prototypicality, socio-political invisibility, and the absence of counterspaces. Overall, our findings highlight unique psychological experiences that exist where multiple subordinate-group identities interact and note the important links between social context and the self.
\end{abstract}

The relationship between the self and its social milieu has long been discussed within socio-psychological theory and research. In particular, scholars have conceptualized the self as an inherently social phenomenon embedded within a socio-cultural and historical context (Way \& Rogers, 2014). Charles Cooley (1903) used the term "looking-glass self" to describe the intricate links between a sense of self and a person's social environment. He argued that the self is an outcome of people's assumptions about how others view them. That is, we see ourselves through the eyes of other people and incorporate other people's views into our selfconcept. So, how might the self develop for individuals who perceive negative regard from those around them

Alex A. Ajayi and Moin Syed, Department of Psychology, University of Minnesota. This document was typeset using Wiernik's (2019) \#prettypreprint template.

Correspondence regarding this article should be addressed to Alex A. Ajayi, Department of Psychology, University of Minnesota, 75 East River Road, Minneapolis, MN 55455, E-mail: ajayi006@umn.edu

\footnotetext{
${ }^{1}$ Generally, this paper adopts the term "sexual minority" to encompass the multiple operationalization of sexual identity, including self-identification (e.g., gay, lesbian, bisexual),
}

or by the broader culture? More specifically, what is the nature of the self as a member of a minority group that, because of its physical or cultural characteristic, is stigmatized, subordinated, and negated? In this chapter, we endeavor to shed light on the links between oppressive social conditions and psychological well-being. While there are many groups who are negatively singled out by others in society for unequal treatment, we explore the experiences of Black sexual minorities ${ }^{1}$ who are simultaneously subjugated to systems of oppression based on their race and sexuality, and focus on the ways in which they may internalize the negative societal attitudes and assumptions about these identities.

Systems of oppression are often considered pernicious and insidious forces that sometimes cause members of marginalized groups to turn oppression inward and become agents of their own oppression (Bulhan, 1985). Pheterson (1990) described this phenomenon of internalized oppression as "the mechanism within an oppressive system for perpetuating domination not

sexual behavior, and sexual attraction - except in the discussion of specific studies where sexual identity is termed differently. 
only by external control but also by building subservience into the minds of the oppressed groups" (p. 35). Given that the self-concept of Black sexual minorities is formed in a socio-cultural context of marked stigma toward both their racial and sexual identities (Greene, 1994), internalized oppression, to some degree, may be inevitable. This is particularly distressing, as scholars have suggested that internalized oppression may be psychologically deleterious to minority group members (Tappan, 2006). Unfortunately, the nature and process by which members of this group may come to internalize and perpetuate such oppression remains largely unexamined in the extant psychological literature.

In this chapter, we propose a theoretical approach, the dual minority stress model, for understanding how socio-cultural stigma "gets under the skin" or is internalized. This model is a confluence of the minority stress theory and intersectionality. The minority stress theory supposes that members from stigmatized groups experience a mismatch between their marginalized identity and the dominant culture (Meyer, 2003). This lack of correspondence is expected to result in a stress experience that is unique, that is, above and beyond the general stressors experienced by those without the stigmatized identity. Intersectionality is a framework used to understand the implications of oppressions that simultaneously operate across multiple dimensions of disadvantage (Cole, 2009). We integrate the minority stress model with intersectionality because Black sexual minorities who identify with both sexual and racial minority groups will likely experience a unique combination of stressors and adaptations related to the concurrent development and articulation of both marginalized identities (Stirratt, Meyer, Ouellette \& Gara, 2007).Over the last five decades, broad societal and economic shifts have had important impacts on how individuals must approach their careers and relate to their employers (Sullivan \& Baruch, 2009). Globalization and rapidly changing technologies have reduced job security and demanded that employees flexibly manage fluid job demands (Hall, 2004; Lepine, Colquitt, \& Erez, 2000; Savickas et al., 2009). Psychological contracts between organizations and employees have become more transactional, leaving individuals less able to rely on their employers for resources, lifelong employment, or opportunities for advancement (Hall, 1996; Sturges, Conway, Guest, \& Liefooghe, 2005). Individuals who are better able to adapt to these unstable circumstances experience better career outcomes (Sullivan \& Baruch, 2009).
We have two goals for this chapter. First, we will outline the dual minority stress model, thereby identifying and elucidating the processes by which culturally oppressive social conditions can create debilitating cognitive, affective, and behavioral patterns wherein oppression is internalized. To date, much of the minority stress research has focused on concrete, prejudicerelated events that occur to the individual, such as the experiences of subtle and overt discrimination among minority individuals. Much less is known about the intangible and internal stigma related processes that happen within the individual, such as minority people's internalization of the social denigration on their minority identity (Meyer, Ouellette, Haile, \& McFarlane, 2011). Given this, the second goal of this chapter is to use narrative methods to flesh out the model and provide empirical support for the more intangible but pervasive component of minority stress: the non-event, intra-individual process of internalized oppression. We will examine the interview narratives of Black sexual minority individuals in order to provide insight on the psychological processes and the social conditions by which oppression is experienced and internalized.

\section{Minority Stress Theory and Internalized Oppres- sion: Contributions and Limitations}

The theory of stress indicates that all social stress results from discordance between the individual and his or her subjective experience of the world (Lazarus \& Folkman, 1984). As an extension of this, minority stress is a consequence of the socio-cultural relegation of particular groups, and it is conceptualized to stem from relatively stable underlying social structures, institutions, and processes (Szymanski, KashubeckWest, \& Meyer, 2008). This ascription of inferiority to these groups, particularly based on identity variables such as race, gender, and sexuality, is posited to result in a stress experience that is ubiquitous and harmful (Brooks, 1981).

This negative social positioning may precipitate negative life events, induce a sense of powerlessness, and lead to maladaptive responses (Meyer, 1995, 2003; Tappan, 2006). While responses to excess stress due to minority status may vary according to mediating individual difference variables and the availability of external and internal resources, minority stress theory indicates that minority stress requires more adaptation than the general stressors experienced by all people. When taken together, minority stress theory can provide a framework for identifying factors relevant to development and adjustment of minority group members, as 
well as the content and process of internalized oppression.

Scholars have long reasoned that stigma and marginalization create stressful social environments that may precede physical and psychological distress among African Americans (Harrell, 2000) and sexual minorities (Brooks, 1981). While not often termed "minority stress," the toll of social oppression has also been empirically examined among African Americans. In particular, perceived racial discrimination has gained increased scholarly attention as a stress process that could have consequences for health and wellbeing. Perceived discrimination is conceptualized as the behavioral manifestation of racism, the organized system (a) underpinned by an ideology of inferiority in which certain racial groups are positioned as culturally inferior to a dominate group and (b) supported by social norms and institutions that implement this ideology (Williams \& Mohammed, 2010). Perceived racial discrimination among African Americans and other racial minorities has been linked to a number of negative physical and psychological outcomes, including psychological distress, increased symptoms of depression, antisocial behaviors, heightened and more negative physiological stress responses, heightened hypertension risk, increased participation in unhealthy behaviors, and decreased participation in healthy behaviors (Ajayi \& Syed, 2014; Pascoe \& Richman, 2009; Williams \& Mohammed, 2010).

Minority stress theory has specifically been used as an explanatory framework for the higher rates of adverse behavioral and mental health outcomes observed among sexual minorities when compared to their heterosexual counterparts, including HIV risk behaviors, substance misuse, psychological distress, and suicides (Hatzenbuehler, 2009; Meyer, 2003). Sexual minority stress theorists have proposed a two-pronged conceptualization of stigma-related stress among this population, comprising distal/external and proximal/internal stress processes (DiPlacido, 1998; Meyer, 1995, 2003). Distal or external stressors in this model include antiLGB harassment, discrimination, and violence; whereas, internal or proximal stressors include expectations of rejection, hiding and concealing of sexual identity, and the internalization of negative societal attitudes (i.e., internalized oppression). These theorists further suggest that individual coping and social support may ameliorate the deleterious effect of minority stress, while relative salience of minority sexual identity may exert moderating influence on various component of this pathway
While sexual minority theorists have presented an elegant theoretical framework for understanding how external and internalized social oppression may have dire implications on physical and mental health, it is not without important shortcomings. First, this framework offers little insight into the process and mechanisms by which individuals come to internalize the negative societal attitudes and assumptions about their minority identity (Szymanski et al., 2008). Moreover, the majority of the empirical research using minority stress theory has focused on sexual minorities from White backgrounds. Therefore, the nature and implications of minority stress is not well known among Black sexual minorities, who are subjugated to crosscutting stressors based on persistent racism and the stigma attached to their sexual identity. To address this limitation, we integrate the minority stress model with intersectionality. The resultant dual minority stress models forces new questions to be examined, such as whether the minority stress process, along with its antecedents and consequences, applies to Black sexual minorities, and whether it explains how members of this group may come to internalize and perpetuate the oppression related their marginalized Black and sexual identities.

\section{Intersectionality and Dual Minority Stress: A Con- comitant Examination of the Content and Process of Internalized Racism and Heterosexism}

With this chapter, we hope to use the dual minority stress model to further the understanding of internalized oppression, which seems to be an important component of the experiences of individuals with marginalized identities. Indeed, much of the existing empirical research on identity-based social oppression has assessed ethnic and sexual identities as singular, homogenous constructs that are relatively isolated from other identities and each other. This approach overlooks the ways in which social identities are simultaneously experienced and misses the unique psychological experiences that exist where identities interact. This has led prominent intersectionality theorists to encourage an intersectional perspective when studying identity and other related psychological processes (Cole, 2009; Syed, 2010; Warner \& Shields, 2013). The intersectionality framework argues that multiple axes of disadvantage interact to influence people's experiences, perceptions, and enactments of self in different contexts (Cole, 2009; Warner \& Shields, 2013). As such, intersectionality can be understood as comprising three important components: "(a) no social group is homogenous, (b) people must be located in terms of social 
structures that capture the power relations implied by those structures, and (c) there are unique, non-additive effects of identifying with more than one social group" (Stewart \& McDermott, 2004, pp. 531 - 532).

By integrating minority stress theory and intersectionality, we hope to shed light on the proximal or internal stress processes that reside at the intersections of race and sexuality. Accordingly, this chapter is guided by two research questions. First, what is the content of internalized oppression for Black sexual minorities? That is, what are the messages being internalized? Second, what is the process by which individuals come to internalize these messages? With this chapter, we hope to expand knowledge about the relationship between the self and an oppressive social environment. We anticipate that the interview narratives of these Black sexual minorities will provide rich contextual information about the construct of internalized oppression and further efforts to attenuate the links between social oppression and well-being.

\section{Method}

\section{Participants and Procedures}

Interview data used in this chapter come from a subsample of participants in a larger qualitative study $(N=$ 30) exploring stress and resilience among sexual minorities. In this chapter, we report on the experiences of 15 participants ( 5 women and 10 men) who identified as sexual minorities and Black (See Table 1). Participants ranged in age from 19 years to 79 years $(M=$ $45.5, S D=23.2$ ). Participants were recruited from three sites serving sexual minorities in New York City and in a medium-sized city in a Mid-Western state using purposeful criterion-based sampling strategies (Creswell, 2013). Sites included community, social, and human services agencies and organizations to ensure a wide diversity of cultural, age, socio-economic, ethnic/racial, and sexual identity representation. A snowball sampling technique was also used (upon the completion of the interview, participants were asked to refer potential participants to the study). Participants were eligible if they were over 18 years old and identified as nonheterosexual.

The study protocol was approved by the university's IRB. The interviews were conducted in private meeting spaces at the recruitment sites or the small Mid-Western university. Upon arriving at the interview location, participants were informed that their participation in the study was voluntary, and each gave informed consent. All participants received a $\$ 10$ gift card for their time. Participants completed a demographic questionnaire, followed by an individual interview using semistructured format with a predefined set of prompting questions. The interviews were audiotape recorded and lasted an average of 45 minutes. Participants were encouraged to speak freely about their lives and experiences during the interview. The current report is based on participant narratives around proximal minority stress, guided by the following questions: (1) Please describe your coming out process. (2) Has there been a situation when you wished you were not your sexual orientation, or when you considered it a shortcoming? (3) What do you think are some of society's perceptions of LGB people? In your opinion, how has this changed over time? (4)Were there certain periods that were easier or more difficult to be LGB? If so, when and how? While the interview questions focused primarily on sexual identity, many of our participants discussed the role their race and ethnicity in their experience of their sexual identity. When participants discussed these connections, they were asked follow-up questions about the intersections of their racial and sexual identities (e.g., can you say more about what it means to you to be gay and Black?).

\section{Data Analysis}

Interview audio recordings were transcribed verbatim and edited to remove identifiers. Transcripts were compared to audio recordings at least twice to ensure the integrity of the transcriptions and read thoroughly several times to become acquainted with the data. A phenomenological framework guided the analysis of the interview data. The goal of phenomenology is to describe "the common meaning for several individuals of their lived experiences" with respect to a particular phenomenon (Creswell, 2013, pp. 76). As such, our analysis sought to develop a composite description of internalized oppression vis-à-vis dual minority stress for Black sexual minorities. Analysis was ongoing throughout the entire study. Interpretive memos were written right after each interview. A research team comprising the first author and 6 undergraduate research assistants wrote extensive case summaries. The case summaries were compared within and between participants for commonalities and distinction with regard to the research questions. Portions of the narratives that provided insight into how participants come to internalize oppression were highlighted and used to develop a "cluster of meaning" (Creswell, 2013), which are the themes presented below. 
AJAYI AND SYED

Table 1

Participant Characteristics

\begin{tabular}{lllll}
\hline Name* & Age & Education & Sexual Identity Label & Racial/Ethnic Identity Label \\
\hline Women & & High School & Lesbian & Black \\
Janet & 83 & Masters & Lesbian & Black \\
Sasha & 79 & Lesbian & Black \\
Rebecca & 75 & Masters & Lesbian & Black \\
Rachel & 70 & Bachelors & Lesbian & Black \\
Teresa & 39 & Bachelors & & \\
& & & & Black \\
Men & & & Gay & Black \\
Shawn & 70 & Some High School & Gay & Black \& Asian \\
Mark & 44 & Masters & Gay & Black, Native American \& Latino \\
Thomas & 42 & Masters & MSM & Black \\
Justin & 32 & Masters & MSM & Black \& White \\
Luke & 30 & Some College & Gay & Black \& Asian \\
Ben & 29 & Some College & Gay & Black \\
Adam & 26 & Masters & Gay & Black \\
Patrick & 23 & Some College & Gay & \\
Francis & 21 & Some College & Gay & \\
Paul & 19 & Some College & & \\
\hline
\end{tabular}

*Pseudonyms are used to protect participants' identity.

\section{Results and Discussion}

We took a phenomenological approach to data analysis in order to identify the core aspects of the participants' experience of internalized oppression at the intersection of their marginalized racial and sexual identities. The narratives reported here provide support for the utility of the dual minority stress model in understanding proximal/internal stress processes that result from crosscutting oppression. The results are organized around our two related overarching research questions: What is the content of internalized oppression for Black sexual minorities? And, what is the process by which negative messages about non-dominant racial and sexual identities become internalized.

\section{"The What": The Content of Internalized Oppres- sion Among Black Sexual Minorities.}

The content of internalized oppression focuses on what this phenomenon looks like. That is, what are the manifestations of internalized oppression for the Black sexual minorities? Our findings point to a multidimensional conceptualization of the content of internalized oppression, comprising an affective component: psychological maladjustment, a behavioral component: acting-out mechanisms, and a cognitive component: identity disintegration. Given that how individuals feel, act, and think about themselves and their identities are interrelated, a major assumption of our conceptualization is that these dimensions of internalized oppression are inescapably joined, each having implications on the others.

Psychological Maladjustment. Scholars have long credited Martinique-born Afro-French psychiatrist, Frantz Fanon, with introducing the phenomenon of internalized oppression in his writings about the consequences and implications of racism and oppression due to colonization on the Black psyche in the 1950s and 1960s (Bulhan, 1985). Over five decades later, Fanon's contemporaries continue to theorize on the psychological impact of oppression and tend to arrive at a common conclusion: the psychological internalization of social systems of prejudice, discrimination, and oppression can lead to a sense of inferiority, low self-esteem, and aggression (Tappan, 2006). Our findings echo some of these postulations. While none of our participants were in acute distress during the interviews, some recounted past emotional strain related to their stigmatized identities. Perhaps most striking is Paul's narrative around difficulties with self-acceptance:

My coming out process was not an easy one. I formally came out to my best friend in the summer of 2008 after fighting depression and suicide tendencies for so long...I am not out to my immediate and extended families because they have vocally said they despise/hate gay people, gay people will die and burn in hell and being gay is un-Black...I cannot stand to see the anger and hurt that will be on their faces. 
Paul's story highlights that our understanding of the tendency for some individuals to turn the stigma-related distress patterns inward cannot be disentangled from their racialized social context. With narratives such as Paul's, it is important to heed the caution against a unilateral representation of all Black families and surely, negative attitudes toward sexual minority identity are not isolated to Black families and communities (e.g., Miller, 2011). However, a number of researchers have brought attention to the unique challenges Black sexual minority individuals encounter in their families and communities (e.g., Greene, 1994; Gutierrez, \& Dworkin, 2003). The experiences of Paul contextualized within this identity politics literature suggest that the intersection of race and sexuality is a fraught domain. Moreover, minority stress theorists have posited that such an expectation of rejection evidenced in Paul's statements often accompany marginalized identities and has been identified as an important minority stressor that leads to a variety of negative psychological outcomes (Meyer, 2003)

Other participants discussed their resilience in spite of race and sexuality related minority stressors. For example, Justin talked at great length about some of the challenges of being gay and a man of color in the United States but also regarded his intersecting identities as co-influencing and source of strength: "I have no problem talking about being gay, but part of the reason why I don't have a problem talking as much about it is that I am comfortable with my own culture. Being Black and Latino, and even, you know, and embracing my Blackness, my Native Americaness, my Latinoness."

Acting-Out Mechanisms. Other participants discussed how multiple axes of oppression impact their self-concept and in turn can lead to self-destructive behavior. Mark described how his "cognitive struggles" related to being Black and gay led to risky sexual behavior: "I was just sex-hunting. I was acting out...but doing it had a lot of shame behind it. I dealt a lot with doing that for many, many years and being very destructive about it to the point of having to get involved in therapy and learning that part of my maybe unhappiness with my life and my depression and things were due to not accepting myself." Similarly, Luke discussed self-destructive behaviors as consequents of non-acceptance and self-hate but also noted that these feelings were not perpetual. "I began to respect gay men as people and not just vessels to fill and as the years went on I began to accept that I'm no different and I began to love and embrace my sexuality." Bulhan (1985) argued that under prolonged oppression, the oppressed may adopt the guidelines and prohibitions that stigmatize their minority identity and as a result, "the oppressed may act out, on each other, the very violence imposed on them. . . . They engage in self-destructive behavior injurious to themselves, their loved ones, and their neighbors" (p. 126).

Identity Disintegration. A number of the participants highlighted the distinctiveness of the Black sexual minority identity and the difficult task of integrating their racial and sexual identities. They discussed the heterosexism in the Black community and racism in LGB community as well as conflicts between the two communities. The hostilities within these communities and conflict between these communities seem to substantially impact the self concept of Black sexual minority individuals. For example, Teresa described the feeling of being caught between two worlds:

When I am in contact with other LGBT people, especially those that are white, the minute I start bringing up the other aspects of who I am, if I bring up race, class and so on, people get very uncomfortable and intimidated. Essentially, it is like a big elephant in the room. The same thing happens when I am around other Black people, the minute I bring up sexuality, it becomes a problem. This is very challenging because I am heavily involved in both communities. It is like you have to pick and choose.

Like Teresa, other participants discussed how their sexual and racial identities seem mutually exclusive; many of them felt like they have to pick a side to align with and in doing so silence their other identity. As previously discussed, many scholars had established the important links between social identity and self-concept. Of note, Icard (1986) argued that some Black gay men experience their sexual and racial identities to be in conflict. He further postulated that racism in the gay community and heterosexism in Black communities may result in Black gay men developing inadequate coping techniques and poor self concepts. Indeed, this postulation is furthered by others who have pointed out the paradox of the Black church, which has served as one of the mainstays of Black resistance to racial oppression, but is one of the most antagonistic to Black non-heterosexuality (Collins, 2004). The conceptualization of identity disintegration as a facet of internalized oppression is rooted in the process of reflective appraisal (see Icard, 1986), which is the process whereby self-concept is based the perception of a group's appraisal. The simultaneous internalization of the negative messages about Blackness from the LGB community and negative messages about non-heterosexuality from the Black community is likely to be characterized 
by feeling that one's identities are in conflict. It is also likely to compromise how Black sexual minorities are able to adaptively respond to the social pressures related to their marginalized identities.

\section{"The How": The Process of Internalized Oppres- sion among Black Sexual Minorities.}

The process of internalized oppression focuses on how this phenomenon comes to be. That is, we refer to the process or mechanism by which individuals come to internalize the social denigration of their minority identity. The process of internalized oppression focuses on the link between the self and its social environment, specifically explicating how social context can exert negative influence on the self. Our findings point to three interrelated processes or dominant societal variables by which stigma becomes internalized: notions of prototypicality about Blackness and non-heterosexuality, socio-political invisibility of the experiences of Black sexual minorities, and the absence of counterspaces that facilitate adaptive responding to oppression.

Notions of Prototypicality. Participants spoke of the essentialist ideologies of race and sexuality. In particular, for some of participants, there was a marked awareness of the norms and standards of Blackness and being non-heterosexual and how these essentialist assumptions tend to distort their subjective realities. Adam describes the prototype of a gay man:

And I think that the idea of the gay man as being urban, as being upper class to upper-middle class who are wealthy. Of being probably under the age of 30 or at least looking like under the age of 30 being white, being fit, being, like all these things that we can think of, you know, who enjoy shopping, who doesn't have a problem with capitalism, who probably hasn't read a nonfiction book ever, there this myth of this person...But you know, I feel like there this model myth and there's everybody else.

In parallel, there seems to be an acknowledgement of White non-heterosexuality among Black communities, but an assumption that Blacks could not be nonheterosexual or that those Blacks who are non-heterosexual are less "authentically" Black because they engage in White sexual practices. As Luke notes, "I live in a predominately Black community. Say if I came in my neighborhood holding hands with another Black gay man. People will be like, "hell no" and we would get disrespected. But if it were two white men, it wouldn't be as much of a problem. Being gay is seen as a White man's disease."
The prominence of these prototypes seems to marginalize individuals who do not fit the model of their constituent group (Purdie-Vaughns \& Eibach, 2008). For Black sexual minorities, the ascription to non-prototypicality functions to "other" their experiences, voices, group membership and in so doing, reinforce White and heterosexual hegemony (Collins, 2004). We argue that the designation of Black sexual minority identity as a problematic deviation from a prototype seems to be a critical impediment to healthy self-concept and esteem. As Brooks (1981) pointed out, "The lexicon of human groups is not a benign classificatory system, but an instrument of communication and control used to define the identity, status, and destiny of target groups" (pp. 57). As such, notions of prototypicality can leave the deep psychological marks of inferiority and deficiency in the psyche of those categorized as deviant.

Francis describes the root of the trepidation that surrounded his sexual identity development and disclosing his sexual identity to his family: "Because, you know, growing up in an African-American family, you know, you always just, you know. That stigma, that Black men go portray this masculine role. You can't be gay, you know. You're not born that way, you know". The psychological toil of rigid cultural narratives about Black sexual identity is further evidenced by Mark's statements about his journey to self-acceptance and positive regard of his concomitant sexual and racial identities. "I had to go through my own process and do a lot of therapy myself to address my own conflicts, my issues, and yes, to start to self-actualize, to accept, to undo and unlearn a lot of concepts that I had around being gay and being Black".

Socio-Political Invisibility. Our findings echo past research that has suggested that oppression at the intersection of race and sexuality among Black sexual minority is wielded in part by cultural scripts that silence their unique voices and perspectives, privileging Black heterosexual and White sexual minorities voices as standard (Purdie-Vaughns \& Eibach, 2008). Participants spoke of the muting or erasure of their perspectives and needs within both Black and mainstream LGBT spaces. For example, Justin spoke of his disillusionment with mainstream LGBT community organizations, which he perceived to have narrow goals that are not always in the best interest of the Black members of this community. In particular, he states:

Oppression based on race is something that is more salient to people of color because it is a minority status that can't be hidden unlike one's sexuality. Everyone in the larger LGBT society wants a piece of the pie, but 
they are leaving LGBT people of color with the crumbs. I don't care as much about gay marriage as I do about the schools in our neighborhoods and so on. There needs to be more coalition building between people of color and LGBT people. For many LGBT people they don't really understand or acknowledge the plight of people of color.

The discussion of the mechanisms by which Black sexual minority come to internalize negative messages about their intersectional experiences and identities would be incomplete without a consideration of the socio-political invisibility of Black sexual minorities. At best, the issues facing Black sexual minorities are often ignored, tokenized or, if they are addressed at all, remain an after-thought or addendum. At worst, their needs and experiences are misrepresented, distorted, and debased.

As the foundation of intersectionality, it is not surprising that Black feminist literature is replete with analyses of Black women's experiences of the intersections of their race and gender and the ways in which multiple axes of oppression are formed and maintain. Moreover, this rich body of work has shed light on the ills of socio-cultural invisibility of Black women within civil rights and feminist movements. In Ain't I a Woman, bell hooks (1981) wrote about the dangerous assumption that all women are White and all Blacks are men, and she challenged the disempowerment, marginalization, and misrepresentation of Black women. Correspondingly, to the extent that cultural schemas erase or distort the experiences of Black sexual minorities, they are likely to be misunderstood by others and misunderstand themselves. In essence, the socio-political invisibility of Black sexual minority experiences in mainstream Black and sexual minority communities functions to not only marginalize but to distort their personhood, the latter which can manifest at the individual level as negative self-concept and stunted identity development. This idea is evident in Mark's recounting of the transformative experience of moving to New York City and for the first time, meeting people who were like him:

It changed my life, you know. I started to embrace more of my sexual orientation, people started to introduce me to what I just thought was...just not available. And one of the things that was unique about it was that...I was able to identify that there were other Black men like myself and I wasn't so unique in my interest in being same-gender loving. So that broke the barriers and I started to self actualize and become more into myself, and embrace the community.
Absence of affirming counterspaces. Our findings highlight the important role of contexts as facilitating or constricting processes related to the development of well-being and health self-concept among Black sexual minorities. Participants spoke about crosscutting factors that contribute to their sense of citizenship and belonging to a community as it relates to well-being. In particular, participants spoke of the need for "counterspaces" that serve as opposites of the "closets" commissioned by systems of racism and heterosexism. Case and Hunter (2012) define counterspaces as "settings, which promote positive self-concepts among marginalized individuals (e.g., racial and sexual minority individuals, persons with disabilities, etc.) through the challenging of deficit-oriented dominant cultural narratives and representations concerning these individuals" (p. 261). As such, counterspaces are sites that promote the psychological well-being of individuals who experience oppression and thus, in contrast, the absence of counterspaces would limit an individual's ability to adaptively cope in the face of oppression.

Indeed, participants discussed the need for safe spaces wherein they could claim, express, and celebrate the complex and interlocking matrices of their multiple identities.

Mark spoke about his sadness regarding the lack of affirming enclaves for Black sexual minorities. "The sadness about that is that more, more, and more of us are coming out. But there are no safe spaces...We'll have more sub-cultural experience where there's high risk behavior, drugs, sex...in a failed attempt to find a space of acceptance where we can find each other". Similarly, Justin spoke about the individual and contextual level processes linked to the growth and enhancement of the Black gay community.

[Black gay men] need a safe space where they can be themselves, a comfortability where they can be actualized and talk about who they are or talk about their sexual experience or be at work and have their male partner on the desk and not have to hide that... to go to primary care medical care wherein they can't talk about their sexual experience as it relates to their medical needs... And having primary care providers and people being insensitive to alternative lifestyles and not having the education or the language to address their needs makes it very unsafe and challenging for Black gay men to live an authentic life... stigma, discrimination continues to increase and impact people's concept about what it means to be gay and what it means to be Black.

These narratives underscore how the intersections of racism and heterosexism results in antagonistic 
mechanisms designed to discipline, isolate, and assault the self-concept of Black sexual minorities because of their socially-constructed deviation from the status quo.

\section{Conclusion}

The advent of intersectionality as an analytical framework represents a paradigm shift in discourse related to issues of power, privilege, oppression, and identity. With this shift comes a growing recognition of the limitation of conceptualizing social categories (such as race, gender, and sexual orientation) as discrete, internally homogenous, and independent. The model of dual minority stress outlined in this chapter attempts to elucidate the links between oppressive social conditions and self-concept among individuals with intersectional marginalized identities. As Black sexual minorities, our participants were able to provide insight on psychological processes and the social conditions by which oppression is experienced and internalized. Specifically, our findings point to three important manifestations of internalized oppression: psychological maladjustment, acting-out mechanisms, and identity disintegration. Our findings also point to three pathways or mechanisms by which negative messages about non-dominant racial and sexual identities become internalized: notions of prototypicality about Blackness and non-heterosexuality, socio-political invisibility of the experiences of Black sexual minorities, and the absence of counterspaces that facilitate adaptive responding to oppression.

This work furthers minority stress theory by examining the stress processes that operate at the junction of multiple axes of disadvantage. Moreover, among the less-studied minority stressors are the nonevent, intangible stressors that occur within the individual, such as the internalization of negative societal attitudes. Our findings provide evidence for the applicability of minority stress theory for Black sexual minorities. As such, we find that internalized oppression is a relevant stress process for this population. However, by employing the dual minority stress framework, our findings also underscore the distinct experience of Black sexual minorities based on the crosscutting stressors related to their racial and sexual identities. The content and process of internalized oppression was linked to a unique amalgam of racism in mainstream American society, racism within the mainstream sexual minority community, negative public regard about sexual minorities, heterosexism within the Black community, the major task of integrating multiple social identities, the erasure and distortion of Black sexual minority experiences, and the lack of safe spaces that affirm their intersecting identities. This understanding would likely have been overlooked by an analytical approach that examines identities as constructs isolated from each other. Our intersectional approach draws attention to how racial and sexual identities are interactively crafted, particularly in the context of stigma and oppression, and the potential for these identity processes to result in internalized negativity.

Our narrative approach also provides insight into the more diffuse and elusive phenomenon of internalized oppression. With the inherent contexualism of qualitative approaches, researchers are better equipped to devise nuanced explanatory models to further understand the complexity of the human experience, particularly the portion of humanity that has historically been ignored or misrepresented. Our findings should implore policymakers and clinicians to give greater consideration to intangible stressors, such as internalized oppression, that may impinge on the lives and well-being of Black sexual minorities. This heightened awareness should also come with interventions and policies that enhance their social citizenship and create counterspaces for them to thrive. 


\section{AJAYI AND SYED}

\section{References}

Ajayi, A. A. \& Syed, M. (2014). Links between patterns of ethnicracial socialization and discrimination experiences and psychological adjustment: A Cluster analysis. Journal of Adolescence, 37(7), 1011-1020.

Brooks, V. R. (1981). Minority stress and lesbian women. Lexington, MA: Lexington Books.

Bulhan, H. A. (1985). Frantz Fanon and the psychology of oppression. New York: Plenum.

Case, A. D., \& Hunter, C. D. (2012). Counterspaces: A unit of analysis for understanding the role of settings in marginalized individuals' adaptive responses to oppression. American journal of community psychology, 50, 257-270.

Cochran, S. D. (2001). Emerging issues in research on lesbians' and gay men's mental health: Does sexual orientation really matter? American Psychologist, 56, 931-947.

Cole, E. R. (2009). Intersectionality and research in psychology. American Psychologist, 64(3), 170-180.

Collins, P. H. (2004) Prisons for our bodies, closets for our minds: Racism, heterosexism, and Black sexuality. In P. H. Collins (Ed.), Black Sexual Politics (pp. 87-116). New York: Routledge,

Cooley, C. H. (1956). Human nature and the social order. New York: Free Press.

Creswell, J.W. (2013). Qualitative inquiry and research design: Choosing among five approaches, ( $3^{\text {rd }}$ Ed.). Thousand Oaks, CA: Sage Publications.

DiPlacido, J. (1998). Minority stress among lesbians, gay men, and bisexuals: A consequence of heterosexism, homophobia, and stigmatization. In G. M. Herek (Ed.) Stigma and sexual orientation: Understanding prejudice against lesbians, gay men, and bisexuals (pp. 138-159). Thousand Oaks, CA: Sage.

Greene, B. (1994). Ethnic-minority lesbians and gay men: mental health and treatment issues. Journal of consulting and clinical psychology, 62(2), 243.

Gutierrez, F. J., \& Dworkin, S. H. (1992). Gay, lesbian, and African American: Managing the integration of identities. In F. J. Gutierrez \& S. H. Dworkin (Eds.), Counseling gay men and lesbians (pp. 141-156). Alexandria, VA: American Association of Counseling and Development.

Harrell, S. (2000). A multidimensional conceptualization of racismrelated stress: Implications for the well-being of people of color. American Journal of Orthopsychiatry, 70, 42-57.

Hatzenbuehler, M. L. (2009). How does sexual minority stigma "get under the skin?" A psychological mediation framework. Psychological Bulletin, 135, 707-730.

hooks, b. (1981). Ain't I a woman: Black women and feminism. Boston, MA: South End.

Icard, L. D. (1986). Black gay men and conflicting social identities: Sexual orientation versus racial identity. Journal of Social Work \& Human Sexuality, 4, 83-93.

Lazarus, R. S., \& Folkman, S. (1984). Stress, appraisal, and coping. New York: Springer.
Meyer, I. H. (1995). Minority stress and mental health in gay men. Journal of Health andSocial Behavior, 36, 38-56.

Meyer, I. H. (2003). Prejudice, social stress, and mental health in lesbian, gay, and bisexual populations: Conceptual issues and research evidence. Psychological Bulletin, 129, 674-697.

Meyer, I. H., Ouellette, S. C., Haile, R., \& McFarlane, T. A. (2011) "We'd be free": narratives of life without homophobia, racism, or sexism. Sexuality Research and Social Policy, 8, 204-214.

Miller, S. J. (2011). African-American lesbian identity management and identity development in the context of family and community. Journal of homosexuality, 58, 547-563.

Pascoe, E. A., \& Smart Richman, L. (2009). Perceived discrimination and health: a meta-analytic review. Psychological Bulletin, 135, 531-554.

Pheterson, G. (1990). Alliances between women: Overcoming internalized oppression and internalized domination. In L. Albrecht \& R. Brewer (Eds.), Bridges of power: Women's multicultural alliances (pp. 34-48). Philadelphia: New Society Publishers.

Purdie-Vaughns, V., \& Eibach, R. P. (2008). Intersectional invisibility: The distinctive advantages and disadvantages of multiple subordinate-group identities. Sex Roles, 59|, 377-391.

Stewart, A. J., \& McDermott, C. (2004). Gender psychology. Annual Review of Psychology,55, $519-544$.

Syed, M. (2010). Disciplinarity and methodology in intersectionality theory and research. American Psychologist, 65, 61-2.

Szymanski, D. M., Kashubeck-West, S., \& Meyer, J. (2008). Internalized Heterosexism A Historical and Theoretical Overview. The Counseling Psychologist, 36, 510-524.

Tappan, M. (2006). Reframing internalized oppression and internalized domination: From the psychological to the sociocultural The Teachers College Record, 108, 2115-2144.

Warner, L. R., \& Shields, S. A. (2013). The intersections of sexuality, gender, and race: Identity research at the crossroads. Sex roles, 68, 803-810

Way, N., \& Rogers, O. (2014). "[T]hey Say Black Men Won't Make It, But I Know I'm Gonna Make It": Ethnic and Racial Identity Development in the Context of Cultural Stereotypes. In K. C. McLean, \& M. Syed (Eds.), The Oxford handbook of identity development (pp. 269-285). New York: Oxford University Press.

Wiernik, B. M. (2019, October 11). Preprint templates. https://doi.org/10.17605/OSF.IO/HSV6A

Williams, D. R., \& Mohammed, S. A. (2009). Discrimination and racial disparities in health: Evidence and needed research. Journal of Behavioral Medicine, 32, 20-47.Abele, A. E., \& Spurk, D. (2009). The longitudinal impact of self-efficacy and career goals on objective and subjective career success. Journal of $\mathrm{VO}$ cational Behavior, 74(1), 53-62. https://doi.org/10/crh36x 